\title{
Clinical Outcomes of Superior Capsular Reconstruction for Massive, Irreparable Rotator Cuff Tears: A Systematic Review Comparing Acellular Dermal Allograft and Autograft Fascia Lata
}

\author{
Tyler J. Smith, D.O., Anirudh K. Gowd, M.D., John Kunkel, D.O., Lisa Kaplin, D.O., \\ John B. Hubbard, M.D., Kevin E. Coates, M.D., Benjamin R. Graves, M.D., and \\ Brian R. Waterman, M.D.
}

\begin{abstract}
Purpose: To investigate clinical outcomes after superior capsular reconstruction (SCR) for the treatment of massive and/ or irreparable rotator cuff tears treated with either allograft or autograft. Methods: Using the Preferred Reporting Items for Systematic Reviews and Meta Analyses (PRISMA) guidelines, in April 2020 a systematic review was performed using PubMed, MEDLINE, EMBASE, and Cochrane databases. Clinical studies were assessed for patient-reported outcomes and range of motion, comparing dermal allografts to fascia lata autografts, with a minimum follow-up of 12 months. Results: A total of 16 clinical studies involving 598 patients (606 shoulders) were included for data analysis, with a weighted mean follow-up of 36.9 months (range 12 to 60 ). Visual analogue scale (VAS) pain scores decreased from 4.0 to $6.9 \mathrm{~mm}$ preoperatively to 0 to $2.5 \mathrm{~mm}$ postoperatively. American Shoulder \& Elbow Surgeons score increased from 20.3 to 54.5 preoperatively to 73.7 to 97.0 postoperatively. Forward flexion increased from $27.0^{\circ}$ to $142.7^{\circ}$ preoperatively to $134.5^{\circ}$ to $167.0^{\circ}$ postoperatively. External rotation increased from $13.2^{\circ}$ to $41.0^{\circ}$ preoperatively to $30.0^{\circ}$ to $59.0^{\circ}$ postoperatively. Acromiohumeral distance increased from 3.4 to $7.1 \mathrm{~mm}$ preoperatively to 6.0 to $9.7 \mathrm{~mm}$ postoperatively. The total rates of complications, graft failure, and revision surgery were $5.6 \%$, $13.9 \%$, and $6.9 \%$, respectively. Conclusions: Irrespective of tissue source, SCR serves as a reasonable joint-preserving option for massive, irreparable rotator cuff tears, with favorable short- to midterm improvements in patient-reported outcomes and range of motion. Level of Evidence: IV, systematic review of level III and IV studies.
\end{abstract}

$\mathbf{T}$ he management of massive or irreparable rotator cuff tears is particularly complex for orthopedic surgeons. Technical challenges include the presence of muscle atrophy, fatty infiltration, scarring or adhesions, myotendinous retraction, tendon inelasticity, superior migration of the humeral head, and ultimately, osteoarthritis. ${ }^{1-5}$ In addition, retear rates of primary repair for

From the Department of Orthopedic Surgery, Philadelphia College of Osteopathic Medicine, Philadelphia, Pennsylvania, U.S.A. (T.J.S., J.K.); and the Department of Orthopedic Surgery, Division of Sports Medicine, Wake Forest University School of Medicine, Winston-Salem, North Carolina, U.S.A. (A.K.G., L.K., J.B.H., K.E.C., B.R.G., B.R.W.).

The authors report the following potential conflicts of interest or sources of funding: B.R.G. reports other, Arthrex, Biomet, Depuy-a Johnson $\theta$ Johnson Company, Exactech, Mitek. K.E.C. reports other, Arthrex; nonfinancial support, Arthroscopy Association of North America. J.B.H. reports other, Abiomed, AnaptysBio, Bluebird Bio, Boston Scientific, Celgene, CRISPR Therapeutics AG, GE Healthcare, Intuitive Surgical Johnson $\theta$ Johnson, Mazor Robotics, Nuvasive, Stryker. B.R.W. reports other AAOS, AOSSM, Arthrex, Arthroscopy, Arthroscopy Association of massive rotator cuff tears have been reported to be as high as $94 \%$ within 36 months. $^{6}$ Treatment options vary and include arthroscopic debridement, tendon transfer, interpositional arthroplasty, subacromial spacer interposition, reverse shoulder arthroplasty (RSA), and superior capsular reconstruction (SCR). RSA has become increasingly popular over the last 2

North America, Elsevier, Kaliber AI, Sparta Science, Vericel, Vivorte. Full ICMJE author disclosure forms are available for this article online, as supplementary material.

Address correspondence to Brian R. Waterman, MD, Department of Orthopaedic Surgery, Wake Forest University School of Medicine, Medical Center Blvd, Winston-Salem, NC 27157-1070,U.S.A. E-mail: brian.r.waterman@ gmail.com

(C) 2020 THE AUTHORS. Published by Elsevier Inc. on behalf of the Arthroscopy Association of North America. This is an open access article under the CC BY-NC-ND license (http://creativecommons.org/licenses/by-nc-nd/4.0/).

2666-061X/20637

https://doi.org/10.1016/j.asmr.2020.09.002 
decades and accounts for nearly half of all shoulder arthroplasty performed today. ${ }^{7}$ However, there are concerns regarding the complication rate of RSA and the associated revision burden, particularly in younger patients. ${ }^{8-10}$

Historically, rotator cuff surgery has focused on reestablishing the tendinous attachment to its footprint. More recently, authors have proposed that the superior capsule may serve as a more integral structure, whereby restoration of superior capsular defects may have a greater impact on subsequent glenohumeral biomechanics. ${ }^{11}$ SCR has become increasingly popular in the United States and Europe for the treatment of massive or irreparable rotator cuff tears. ${ }^{12}$ However, the justification for its exponential growth has been questioned, as the literature regarding SCR is limited to several small case series and biomechanical studies. The purpose of this study was to investigate clinical outcomes after SCR for the treatment of massive or irreparable rotator cuff tears treated with either allograft or autograft. The authors hypothesized that postoperative clinical outcomes would demonstrate pain reduction, improved shoulder function, and improved range of motion, and that SCR with fascia lata grafts would demonstrate outcomes superior to those of dermal matrix grafts.

\section{Methods}

\section{Literature Search}

A systematic review was registered with PROSPERO and performed using Preferred Reporting Items for Systematic Reviews and Meta-Analyses (PRISMA) guidelines. A comprehensive literature search was performed using PubMed, Medline (Ovid), EMBASE (Elsevier), and Cochrane Library (Wiley) electronic databases in April 2020. MeSH terms were used to increase sensitivity. Additionally, all references in the included studies were cross-referenced for inclusion if any were missed by the initial search. The final search was completed on April 10, 2020, independently, by 2 authors (T.J.S., L.K.).

\section{Study Eligibility}

Trials were eligible for inclusion if they met the following criteria: human or cadaveric subjects with documented massive or irreparable rotator cuff tears, superior capsular reconstruction, and patient outcome after $\geq 12$ months of follow-up for clinical trials. Studies involving animals, partial rotator cuff tear, rotator cuff repair, operative technique articles without reported outcomes, cadaver studies, biomechanical studies, review articles, comments, letters, editorials, duplicates, and nonrelevant studies were excluded. Case reports, abstracts without available full text, and foreign language articles without direct translation were excluded.

\section{Study Selection and Data Abstraction}

A full-text review was performed by 2 authors (T.J.S., L.K.) to confirm appropriateness for inclusion. Any disagreement between authors during each step of the review process was resolved by discussion. If consensus could not be reached, final inclusion was decided by a third reviewer (B.R.W.). A flow diagram outlining the selection process can be found in Fig 1 .

Clinical studies were assessed for multiple outcomes of interest, including level of evidence, concomitant procedures, visual analogue scale (VAS), Constant score, Oxford Shoulder Score (OSS), UCLA shoulder score, American Shoulder \& Elbow Surgeons score (ASES), Subjective Shoulder Value (SSV), acromial humeral distance (AHD), range of motion (ROM), patient satisfaction, complications, reoperations, revision surgery, conversion to reverse shoulder arthroplasty, and graft failure. Graft failure was defined as retear confirmed by magnetic resonance imaging (MRI) or ultrasound, partially healed graft confirmed by MRI or ultrasound at final radiologic follow-up, or loss of graft fixation resulting in functional deficit. Descriptive statistics were calculated from each included study. For continuous data, weighted means and standard deviations were calculated for all subjects and outcome parameters. Clinical studies were assessed for patient-reported outcomes and ROM in comparison of fascia lata autografts to acellular dermal allografts.

\section{Data Analysis}

The Metafor package as part of RStudio software version 1.0.143 (R Foundation for Statistical Computing, Vienna, Austria) was used for data analysis. Forest plots were created for VAS, ASES, and ROM (forward flexion [FF] and external rotation [ER]) (Figs 2, 3, 4, and 5, respectively). The $I^{2}$ index was used to measure heterogeneity of included studies. ${ }^{13}$ Effect sizes were calculated using random-effects models with the DerSimonian-Laird estimator, as high heterogeneity precluded use of a fixed-effects model. ${ }^{14,15}$

\section{Risk of Bias Assessment}

A funnel plot was created to assess publication bias. Estimated treatment effect for the change in ASES score was plotted on the $\mathrm{x}$ axis, and effect sizes were plotted on the y axis. ${ }^{16}$ Study methodological quality was assessed independently by 2 authors (T.J.S., J.K.) using the Methodological Index for Non-randomized Studies (MINORS) score (Table 1; Appendix).

\section{Results}

\section{Study Identification and Assessment}

The following terms were used as keywords and appeared in the title, abstract, or keyword fields: (1) massive rotator cuff tear $(\mathrm{n}=463)$; (2) irreparable rotator cuff tear $(\mathrm{n}=278)$; (3) superior capsular 
Fig 1. PRISMA (Preferred Reporting Items for Systematic Reviews and Meta-Analyses) flow chart.
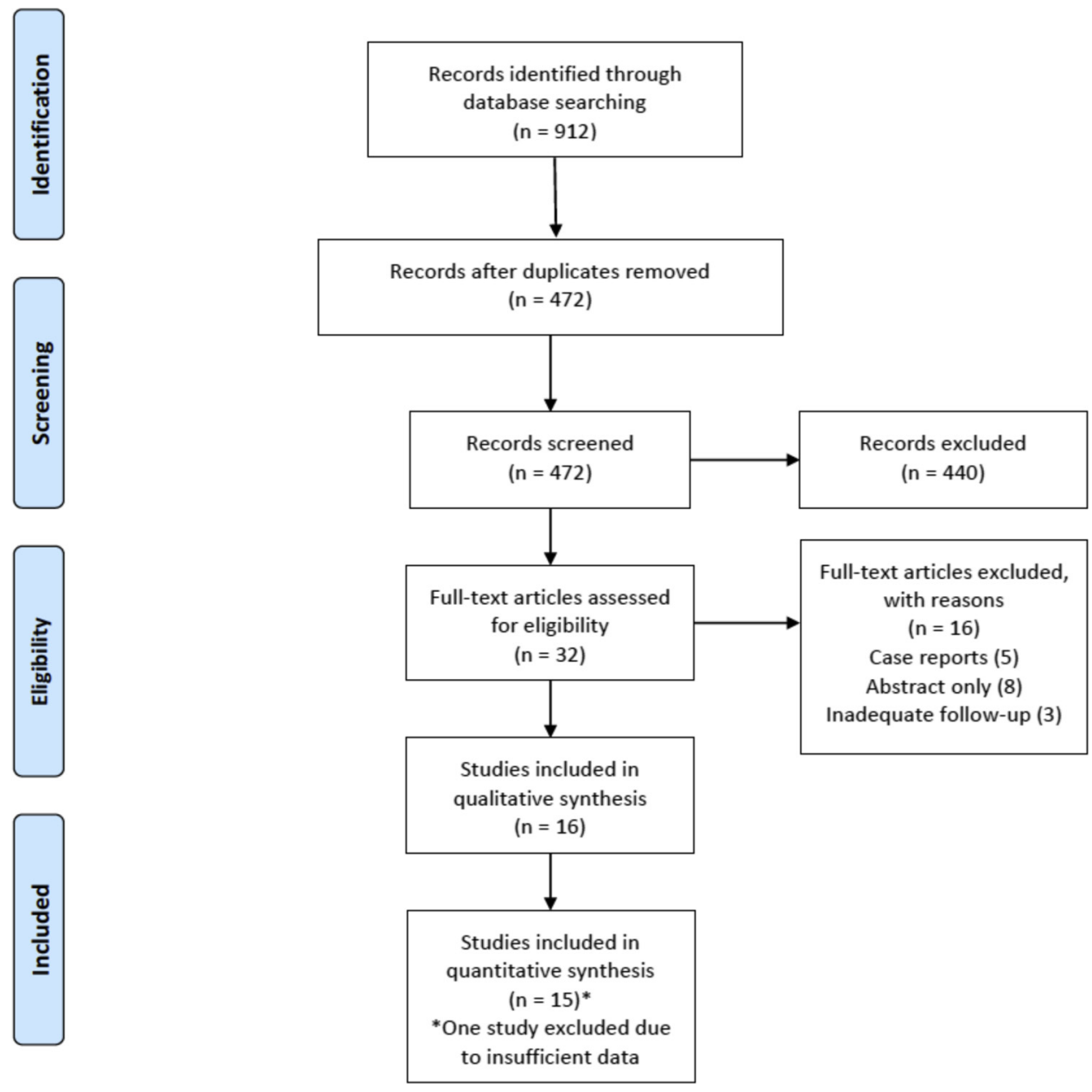

reconstruction ( $\mathrm{n}=98$ ); and (4) superior capsule reconstruction $(\mathrm{n}=73)$. The initial keyword search returned 912 articles for review. After screening for duplicate citations, 472 articles remained. After screening for appropriateness based on the title and abstract, 440 articles were excluded, leaving 32 articles

\section{Change in VAS}

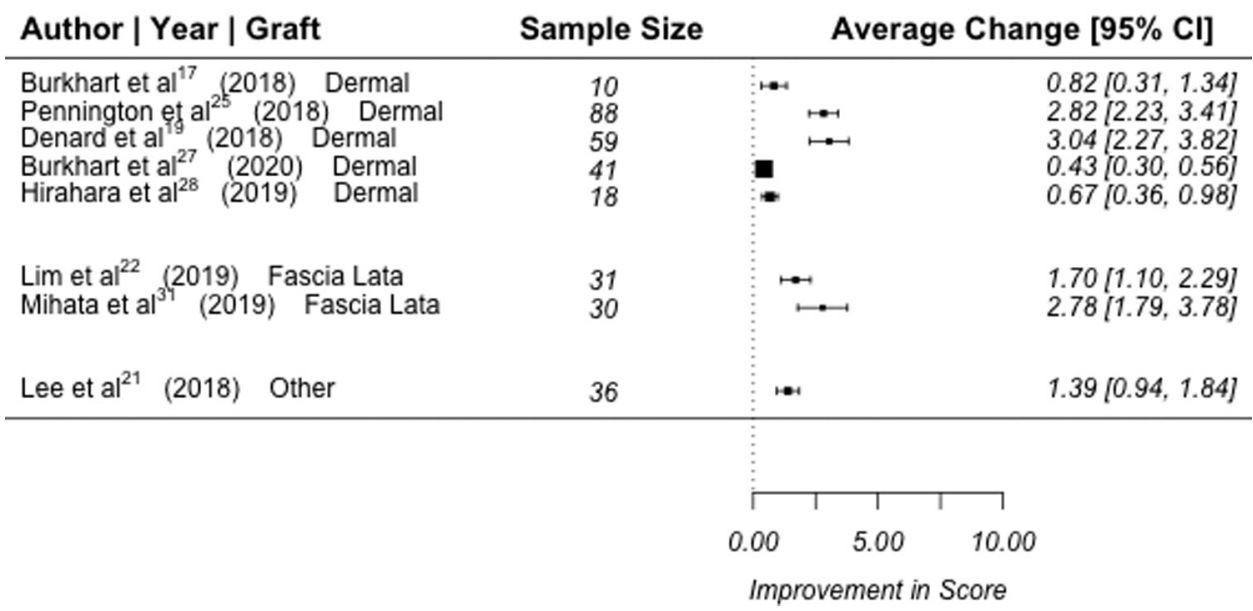

Fig 2. Forest plot demonstrating change in visual analogue scale (VAS) after superior capsular reconstruction (SCR) with dermal matrix versus fascia lata grafts. Abbreviation: CI confidence interval. 


\section{Change in ASES}

\section{Author | Year | Graft}

Burkhart et $\mathrm{al}^{17}$ (2018) Dermal

Pennington et al ${ }^{25}$ (2018) Dermal

Denard et $\mathrm{al}^{19}$ (2018) Dermal

Hirahara et al ${ }^{20}$ (2017) Dermal

Burkhart et $\mathrm{al}^{27} \quad$ (2020) Dermal

Hirahara et al ${ }^{28}$ (2019) Dermal

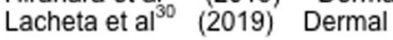

Mihata et $\mathrm{al}^{12} \quad$ (2013) Fascia Lata

Lim et al ${ }^{22}$ (2019) Fascia Lata

Mihata et $\mathrm{al}^{23}$ (2018) Fascia Lata

Mihata et $\mathrm{al}^{24}$ (2018) Fascia Lata

Mihata et $\mathrm{al}^{31}$ (2019) Fascia Lata

Lee et $\mathrm{al}^{21}$ (2018) Other

\section{Sample Size}

$\begin{array}{llr}8 & 6.71[2.06,11.36] \\ 88 & & 21.83[17.27,26.39] \\ 59 & 17.81[21.46,36.16] \\ 8 & & 17.96[5.51,30.41] \\ 41 & & 5.13[3.56,6.70] \\ 18 & & 4.94[2.66,7.22] \\ 22 & & 23.11[13.45,32.76]\end{array}$

$18.30[10.98,25.63]$

$20.91[13.55,28.26]$

$21.63[17.39,25.87]$

$18.18[14.38,21.98]$

$22.50[14.45,30.55]$
Fig 3. Forest plot demonstrating change in American Shoulder \& Elbow Surgeons Score (ASES) after superior capsular reconstruction (SCR) with dermal matrix versus fascia lata grafts. Abbreviation: CI confidence interval. for full text review. Of those 32,5 were case reports, 8 were abstracts without available full text, and 3 did not meet the minimum follow-up. Sixteen studies, all level of evidence III or IV, reporting clinical outcomes were included in the final review for data extraction and analysis (Fig 1). ${ }^{12,17-31}$ All studies lacked randomization or a control group. The mean MINORS score for all studies included was 12.4 (Table 1; Appendix). A funnel plot was created to assess publication bias. Estimated treatment effect for the ASES were plotted on the $\mathrm{x}$ axis, and effect sizes were plotted on the $\mathrm{y}$ axis. Point estimates were verified to be symmetric around the real estimated treatment effect to demonstrate limited publication. However, 7 studies were outside the funnel, which would suggest heterogeneity in results (Fig 6). ${ }^{16}$

\section{Change in FF}

\section{Author | Year | Graft}

Burkhart et al 17| 2018| Dermal
Burkhart et al 27| 2020| Dermal

Fig 4. Forest plot demonstrating change in forward flexion (FF) after superior capsular reconstruction (SCR) with dermal matrix versus fascia lata grafts. Abbreviation: CI confidence interval.

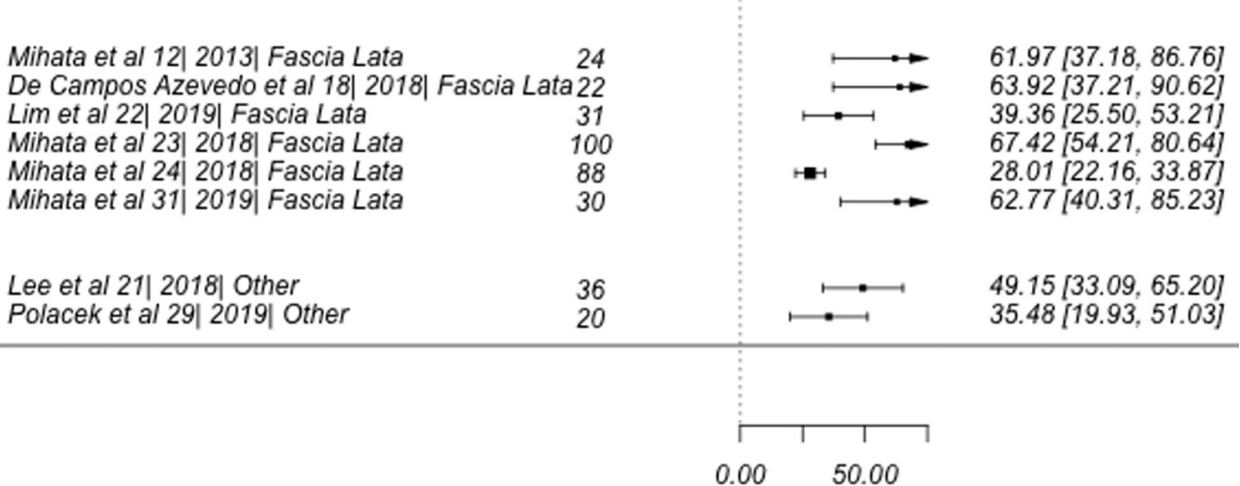

Improvement in Score 
Change in ER

Fig 5. Forest plot demonstrating change in external rotation (ER) after superior capsular reconstruction (SCR) with dermal matrix versus fascia lata grafts. Abbreviation: CI confidence interval.

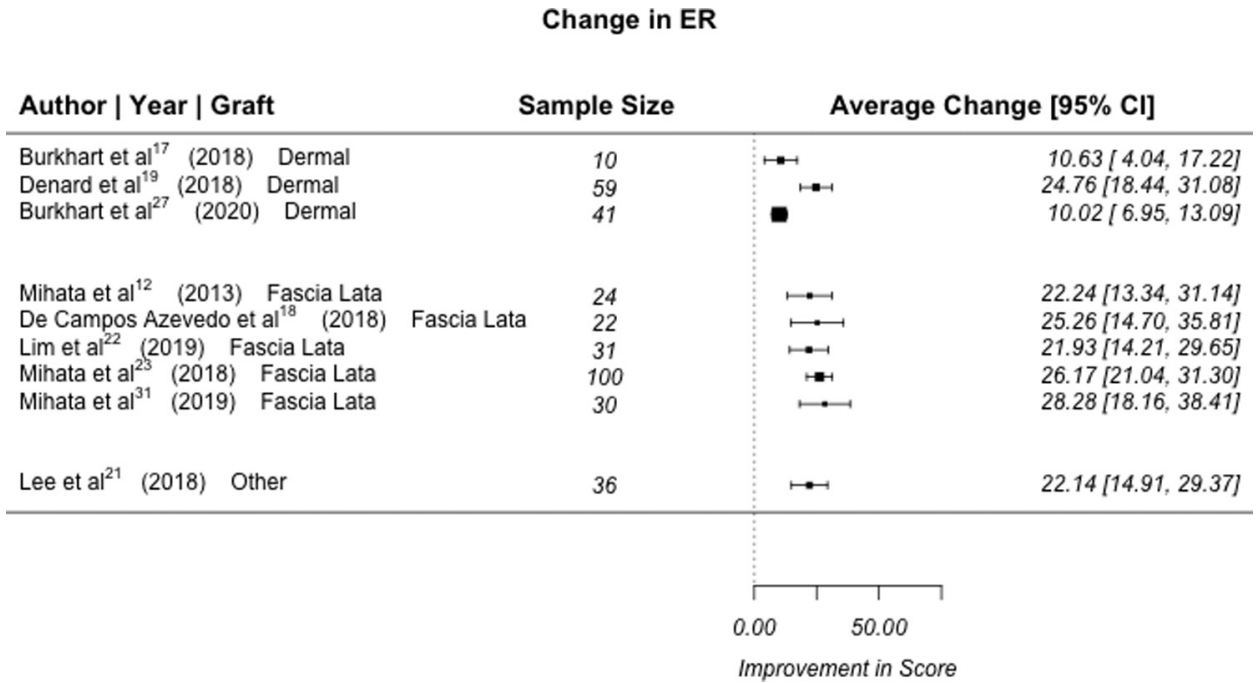

\section{Study and Patient Characteristics}

Of the 16 clinical studies, 6 involved fascia lata autografts, ${ }^{12,18,22-24,31} 8$ involved acellular dermal matrix allografts, ${ }^{17,19,20,25,27-30} 1$ involved both fascia lata and dermal matrix grafts, ${ }^{21}$ and 1 study involved autologous hamstring grafts. ${ }^{26}$ In total, 606 shoulders $(598$ patients) treated with superior capsular reconstruction were pooled for evaluation, with a weighted mean follow-up of 36.9 months (range 12 to 60 ). Graft types were fascia lata in 294 patients $(49.2 \%)$, dermal matrix in $264(44.1 \%)$, hamstring autograft in $8(1.3 \%)$, and not disclosed in $32(5.4 \%)$. Graft thickness was a minimum of $5 \mathrm{~mm}$ (range 5 to 8 ) in 294 patients $(49.2 \%$ ), 1 to $3.5 \mathrm{~mm}$ in $264(44.1 \%)$, and not disclosed in 40 $(6.7 \%)$. All of the $5-$ to $8-\mathrm{mm}$ grafts were fascia lata grafts, and all of the 1- to $3.5-\mathrm{mm}$ grafts were dermal matrix grafts. The mean follow-up by graft type was $46.8,24.2$, and 12 months for fascia lata, dermal matrix, and hamstring grafts, respectively.

\section{Clinical Outcomes}

After SCR, the standard mean difference between postoperative and preoperative state was demonstrated with respect to the VAS score, ASES score, FF, and ER (Figs 2, 3, 4, and 5, respectively). VAS scores were reported in 10 studies, and all studies reported improvements from preoperative (range 4.0 to $6.9 \mathrm{~mm}$ ) to postoperative (range 0 to $2.5 \mathrm{~mm}$ ) values. ${ }^{17,19,20-22,25,27,28,30,31}$ ASES scores were reported in 13 studies, and all studies reported improvements from preoperative (range 20.3 to 54.5 ) to postoperative (range 73.7 to 97.0 ) values. ${ }^{12,17,19-25,27,28,30,31}$ The change in FF was reported in 12 studies, with all studies reporting improvements from preoperative (range $27.0^{\circ}$ to $142.7^{\circ}$ ) to postoperative (range $134.5^{\circ}$ to $167.0^{\circ}$ ) values. ${ }^{12,17-19,21-24,27,29,30,31}$ The change in ER was reported in 10 studies, with all studies reporting improvements from preoperative (range $13.2^{\circ}$ to $41.0^{\circ}$ ) to postoperative (range $30.0^{\circ}$ to $59.0^{\circ}$ ) values. $^{12,17-19,21-23,27,30,31}$ Constant scores were reported in 4 studies, all demonstrating improvements from preoperative (range 17.5 to 56.3 ) to postoperative (range 63.7 to 83.5 ) values. ${ }^{18,21,22,26}$ Japanese Orthopaedic Association scores were reported in 3 studies, all demonstrating improvements from preoperative (range 40.6 to 61.2 ) to postoperative (range 90.6 to 95.2) values. $^{12,24,31}$ AHD was reported in 12 studies, and demonstrated improvements from preoperative (range 3.4 to $7.1 \mathrm{~mm}$ ) to postoperative (range 6.0 to $9.7 \mathrm{~mm}$ ) values in 11 studies. ${ }^{12,18-22,25-27,30,31}$ One study demonstrated a decreased mean AHD postoperatively; however, this finding was not statistically significant $(P=.6){ }^{17}$

\section{Clinical Outcomes by Graft Type}

Clinical studies were differentiated by graft type for subgroup analysis. One study using both fascia lata and dermal matrix grafts did not differentiate outcomes for each subgroup and was therefore excluded. ${ }^{21}$ For SCR with dermal allografts, mean VAS improved from 4.0 to $6.3 \mathrm{~mm}$ preoperatively to 0 to $1.7 \mathrm{~mm}$ postoperatively, mean ASES 41.8 to 54.0 versus 73.7 to 92.3 , mean FF from $27.0^{\circ}$ to $140.0^{\circ}$ versus $137.0^{\circ}$ to $167.0^{\circ}$, mean ER from $24.0^{\circ}$ to $41.0^{\circ}$ versus $35.0^{\circ}$ to $59.0^{\circ}$, and mean AHD from 3.4 to $7.1 \mathrm{~mm}$ versus 6.0 to $9.7 \mathrm{~mm}$. For SCR with fascia lata autografts, mean VAS improved from 6.0 to $6.9 \mathrm{~mm}$ preoperatively to 0.9 to $2.5 \mathrm{~mm}$ postoperatively, mean ASES from 20.3 to 54.4 versus 77.5 to 97.0 , mean FF from $36.7^{\circ}$ to $142.7^{\circ}$ versus $143.8^{\circ}$ to $163.6^{\circ}$, mean ER from $13.2^{\circ}$ to $32.0^{\circ}$ versus $30.0^{\circ}$ to $44.0^{\circ}$, and mean AHD from 4.6 to 6.4 $\mathrm{mm}$ versus 6.4 to $8.7 \mathrm{~mm}$. 
Table 1. Study Demographic Characteristics and Design

\begin{tabular}{|c|c|c|c|c|c|}
\hline Reference & Patients/Shoulders & $\begin{array}{c}\text { Mean } \\
\text { Age }(y)\end{array}$ & $\begin{array}{c}\text { Mean Follow-Up } \\
(\mathrm{mo})\end{array}$ & Study Design/Methodology & $\begin{array}{c}\text { MINORS } \\
\text { Score } \\
\end{array}$ \\
\hline Mihata et al. $2013^{12}$ & $23 / 24$ & 65.1 & 34.1 & $\begin{array}{l}\text { - Retrospective case series (level IV) } \\
\text { - Comparison between retear }(+) \text { and retear }(-) \text { groups } \\
\text { - No adjustment of confounding variables } \\
\text { - No control group }\end{array}$ & $12 / 16$ \\
\hline $\begin{array}{l}\text { Burkhardt and } \\
\text { Hartzler } 2019^{17}\end{array}$ & $10 / 10$ & 69 & 12.9 & $\begin{array}{l}\text { - Retrospective case series (level IV) } \\
\text { - Power analysis included } \\
\text { - No control/comparison groups } \\
\text { - No adjustment of confounding variables }\end{array}$ & $14 / 16$ \\
\hline $\begin{array}{l}\text { Rosales-Varo et al. } \\
2019^{26}\end{array}$ & $8 / 8$ & 59.6 & 12 & $\begin{array}{l}\text { - Prospective case series (level IV) } \\
\text { - No control/comparison groups } \\
\text { - No adjustment of confounding variables }\end{array}$ & $12 / 16$ \\
\hline $\begin{array}{l}\text { Pennington et al. } \\
2019^{25}\end{array}$ & $86 / 88$ & 59.4 & $\begin{array}{l}16 \text { to } 28, \text { no } \\
\text { mean reported }\end{array}$ & $\begin{array}{l}\text { - Retrospective case series (level IV) } \\
\text { - No control/comparison groups } \\
\text { - No adjustment of confounding variables }\end{array}$ & $11 / 16$ \\
\hline $\begin{array}{l}\text { de Campos Azevedo } \\
\text { et al. } 2018^{18}\end{array}$ & $22 / 22$ & 64.8 & $\begin{array}{r}\text { Minimum } 24, \text { no } \\
\text { mean reported }\end{array}$ & $\begin{array}{l}\text { - Prospective case series (level IV) } \\
\text { - Multiple subgroup comparisons included } \\
\text { - Power analysis included } \\
\text { - No adjustment of confounding variables } \\
\text { - No control group }\end{array}$ & $14 / 16$ \\
\hline Denard et al. $2018^{19}$ & $59 / 59$ & 62 & 17.7 & $\begin{array}{l}\text { - Prospective case series (level IV) } \\
\text { - No control/comparison groups } \\
\text { - No adjustment of confounding variables }\end{array}$ & $10 / 16$ \\
\hline Mihata et al. $2018^{24}$ & $88 / 88$ & 65.3 & 60 & $\begin{array}{l}\text { - Retrospective case series (level IV) } \\
\text { - No control/comparison groups } \\
\text { - No adjustment of confounding variables }\end{array}$ & $10 / 16$ \\
\hline Mihata et al. $2018^{23}$ & $100 / 100$ & 66.9 & 48 & $\begin{array}{l}\text { - Retrospective comparison study (level III) } \\
\text { - Comparison between subgroups } \\
\text { - No adjustment of confounding variables } \\
\text { - No control group }\end{array}$ & $12 / 16$ \\
\hline $\begin{array}{l}\text { Burkhart et al. } \\
2020^{27}\end{array}$ & $41 / 41$ & 64 & 34 & $\begin{array}{l}\text { - Retrospective case series (level IV) } \\
\text { - Subgroup comparisons between } 12 \text { mo and } 24 \text { mo } \\
\text { follow-up } \\
\text { - No adjustment of confounding variables } \\
\text { - No control }\end{array}$ & $13 / 16$ \\
\hline $\begin{array}{l}\text { Hirahara et al. } \\
2019^{28}\end{array}$ & $18 / 18$ & 63 & $\begin{array}{l}\text { Minimum } 12 \text {, no } \\
\text { mean reported }\end{array}$ & $\begin{array}{l}\text { - Retrospective case series (level IV) } \\
\text { - Subgroup comparisons between ultrasounds performed } \\
<6 \text { and }>12 \text { mo } \\
\text { - No adjustment of confounding variables } \\
\text { - No control }\end{array}$ & $13 / 16$ \\
\hline Polacek $2019^{29}$ & $19 / 20$ & 60 & $\begin{array}{l}\text { Minimum } 12 \text {, no } \\
\text { mean reported }\end{array}$ & $\begin{array}{l}\text { - Prospective case series (level IV) } \\
\text { - Subgroup comparisons between 6- and 12-mo } \\
\text { follow-up } \\
\text { - No adjustment of confounding variables } \\
\text { - No control }\end{array}$ & $14 / 16$ \\
\hline
\end{tabular}


Table 1. Continued

\begin{tabular}{|c|c|c|c|c|c|}
\hline Reference & Patients/Shoulders & $\begin{array}{c}\text { Mean } \\
\text { Age }(y)\end{array}$ & $\begin{array}{l}\text { Mean Follow-Up } \\
(\mathrm{mo})\end{array}$ & Study Design/Methodology & $\begin{array}{l}\text { MINORS } \\
\text { Score }\end{array}$ \\
\hline$\overline{\text { Lacheta et al. } 2020^{30}}$ & $22 / 22$ & 56 & 25 & $\begin{array}{l}\text { - Prospective case series (level IV) } \\
\text { - Subgroup comparisons between retear }(+) \text { and } \\
\text { retear }(-) \\
\text { - Subgroup comparisons between patients with/without } \\
\text { prior RTC repair } \\
\text { - Power analysis included } \\
\text { - No adjustment of confounding variables } \\
\text { - No control }\end{array}$ & $14 / 16$ \\
\hline Mihata et al. $2019^{31}$ & $30 / 30$ & 68 & $\begin{array}{l}\text { Minimum } 60 \text {, no } \\
\text { mean reported }\end{array}$ & $\begin{array}{l}\text { - Retrospective case series (level IV) } \\
\text { - Subgroup comparisons between retear }(+) \text { and } \\
\text { retear }(-) \\
\text { - Subgroup comparisons between 1- and 5-y follow-up } \\
\text { - No adjustment of confounding variables } \\
\text { - No control }\end{array}$ & $12 / 16$ \\
\hline
\end{tabular}

RTC, rotator cuff.

\section{Complications, Reoperations, and Graft Failures}

From all cases of SCR, there were 82 incidences $(13.9 \%)$ of retear, loss of fixation, or partially healed graft at final radiologic follow-up (range $0 \%$ to $47.6 \%$ ). Of the 82 reported failures, 53 reports included the site of retear or graft failure. Failure occurred on the humeral side in 37 cases $(69.8 \%)$, intrasubstance in 9 cases $(16.9 \%)$, and at the glenoid fixation in 7 cases $(13.2 \%)$. There were 42 revision surgeries performed $(6.9 \%$, range $0 \%$ to $36.1 \%$; 19 revision SCR, 11 RSA, and 2 balloon spacers). No studies disclosed whether grafts were reused during revision SCR procedures. There were 34 complications among all surgeries $(5.6 \%$, range $0 \%$ to $15 \%$ ) (Table 2$)$.

\section{Discussion}

This systematic review suggests that SCR for massive or irreparable rotator cuff tears produces favorable short-term outcomes, with resultant improvements in pain, ROM, and other patient-reported outcomes, irrespective of graft type. In addition, the findings demonstrated low rates of SCR graft failure $(13.9 \%)$, complications $(5.6 \%)$, and revision surgeries $(6.9 \%)$ at a mean follow-up of $>3$ years. These outcomes are especially encouraging given the reported retear rates after primary repair of massive, irreparable rotator cuff tears being as high as $94 \% .^{6}$ By comparison, the postoperative complication rate of RSA has been reported to be as high as 39\%, with increasing rates among younger patient groups. ${ }^{10,32}$

Recently published systematic reviews have echoed these findings, reporting short-term improvements after SCR in both subjective and objective outcomes, including significant improvements in forward elevation and ER. ${ }^{12,33,34}$ Catapano et al. ${ }^{35}$ published a qualitative review of 10 clinical studies in 2019. They reported improvements in VAS, ASES, FF, and ER, with a retear rate ranging from $3.4 \%$ to $36.1 \%$. However, that review included 2 studies that were excluded from our review (1 case report, 1 abstract only). ${ }^{36,37}$ Galvin et al. $^{33}$ published a qualitative review of 5 clinical studies and 5 biomechanical SCR studies in 2019 and reported mean improvements in VAS, ROM, and patient-reported outcomes. Sochacki et al. ${ }^{34}$ published a systematic review of SCR clinical studies in 2019 and showed statistically significant improvements in VAS, ASES, FF, and ER. The average complication, reoperation, and graft failure rates were $3.8 \%, 11.7 \%$, and $14.2 \%$, respectively. However, that review involved only 6 clinical studies, one of which was a case report. $^{19-21,23,25,38}$

\section{Graft Type and Thickness}

The native superior capsule ranges in thickness from 4.4 to $9.1 \mathrm{~mm}^{39}$ Mihata et al. ${ }^{12}$ initially described SCR with a folded, 6- to 8-mm fascia lata autograft. However, concerns of donor site morbidity, shorter operative duration, and ease of graft preparation have led authors in North America to primarily use thinner, 1 - to 3-mm commercially available dermal allografts. $^{17,19,20,25}$ Until recently, few studies have compared the 2 most popular graft types. Galvin et al., ${ }^{33}$ in the qualitative review of 5 clinical and 5 cadaveric studies, suggested improved glenohumeral stability and decreased subacromial contact pressures with 8-mm fascia lata grafts compared with 4-mm acellular dermal allografts. This conclusion was drawn mainly from the biomechanical cadaveric studies by Mihata and colleagues, ${ }^{40,41}$ which demonstrate improved restoration of glenohumeral mechanics, decreased thinning, and decreased elongation with a thicker graft. In their recent systematic review, Makovicka et al. ${ }^{42}$ described SCR outcomes among various graft types in 9 clinical and 8 biomechanical studies. They reported improvements in superior humeral 


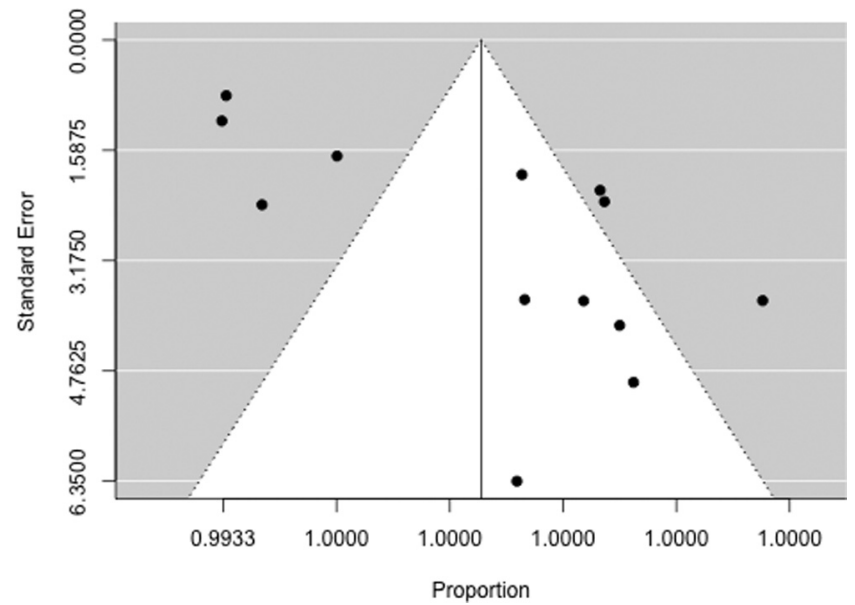

Fig 6. A funnel plot was created to assess publication bias. Estimated treatment effect for the American Shoulder \& Elbow Surgeons Score (ASES) were plotted on the $\mathrm{x}$ axis, and effect sizes were plotted on the y axis. Point estimates were verified to be symmetric around the real estimated treatment effect to demonstrate limited publication bias; however, 7 studies were outside the funnel, which would suggest heterogeneity in results.

translation, subacromial forces, FF, ER, and patientreported outcomes. However no direct, quantitative comparison among graft types was performed in that review. ${ }^{42}$ Interestingly, our analysis of the available clinical data comparing fascia lata autografts and dermal allografts shows similar improvements in VAS, ASES, FF, and ER between these grafts. Despite differences found in biomechanical studies, either graft may be an acceptable option for SCR, with favorable short-term improvements in pain, ROM, and patient-reported outcome scores.

\section{Graft Failures}

A common debate when discussing graft types in SCR is the difference in failure rate. Previous studies have suggested that grafts thicker than $3 \mathrm{~mm}$ outperform thinner grafts, with lower failure rates. ${ }^{19,20}$ Lee et al. ${ }^{21}$ reported that grafts $>6 \mathrm{~mm}$ were more likely to restore the AHD, demonstrating a statistically significant association with graft healing $(P<.04)$. We found the rate of graft failure to be higher in dermal allografts versus fascia lata grafts $(16.7 \%$ versus $9.2 \%$, respectively). However, there was considerable variation in the 16 clinical studies, with 6 studies reporting failure rates of $30 \%$ or greater. ${ }^{17,20-22,29,30}$ In contrast, Mihata and colleagues $^{23,24}$ reported a failure rate of 4 of $24(16.6 \%)$ in their initial 2013 case series, ${ }^{12}$ followed by 9 of 188 $(4.8 \%)$ in subsequent 2018 studies. These findings suggest that there may be a significant learning curve associated with SCR.

When SCR graft failure did occur, it happened most commonly at the site of humeral fixation (69.8\%). Hirahara et al. ${ }^{28}$ noted that as imaged by ultrasound, dermal allografts are thickened and highly vascularized laterally at the humeral fixation site. The authors hypothesized that humeral tension and fixation are crucial factors in regard to graft healing. Similarly, Catapano et al. ${ }^{35}$ reported that $76.7 \%$ of graft failures in their review occurred at the humeral fixation site. These findings may be related to (1) greater tensile forces on the humeral fixation site during glenohumeral ROM and (2) acromiohumeral contact abrasion. ${ }^{19}$ However, it should be noted that 1 study included in the present review that reported 13 of 36 failures, all occurring on the humeral side, performed graft fixation with a single-row technique. ${ }^{21}$ Conversely, other authors have suggested that graft failure occurs most commonly at the glenoid fixation or midsubstance. ${ }^{30,43,44,45}$ Additional clinical studies with long-term follow-up data are needed to determine ideal graft tensioning, as well as to assess whether there are significant clinical manifestations of the elongation and thinning of the dermal allograft as observed in biomechanical studies. Interestingly, Polacek ${ }^{29}$ reported a $15 \%$ rate of early graft failure secondary to immunologic rejection of the dermal allograft. All patients had underlying immunologic disorders and experienced graft disintegration within several weeks of surgery. The optimal graft choice for SCR in patients with autoimmune or immunologic conditions remains unclear.

The merits of this systematic review include its large sample size, with 606 shoulders in 598 patients. In addition, the 16 included studies involve a variety of graft types (fascia lata autograft, acellular dermal matrix, and hamstring autograft) and a variety of graft thicknesses (range 1 to $8 \mathrm{~mm}$ ). This review also includes a subjective synthesis of outcomes between graft types, which has not been analyzed in prior reviews on this topic. In addition, although previous reviews have demonstrated only moderate levels of heterogeneity, the mean MINORS score for our pooled clinical studies demonstrated a high level of methodological quality as well as a high degree of interobserver reliability.

\section{Limitations}

We acknowledge that our analysis has several limitations. First, evaluation of ROM varied between studies, with some authors using a goniometer and others reporting values from visual inspection. In addition, ER was primarily measured with the arm at the side; however, several studies reporting ER did not specify arm position. ${ }^{17,22,27}$ Second, all of the articles included are level of evidence III and IV, and although several studies performed subgroup comparisons, no studies involved a control group or randomization. In addition, many studies failed to control for confounding patient and/or procedural variables such as preoperative shoulder function and concomitant surgeries. Third, 7 of the included studies also demonstrated heterogeneity among results during the risk of bias 
Table 2. Outcomes after superior capsular reconstruction

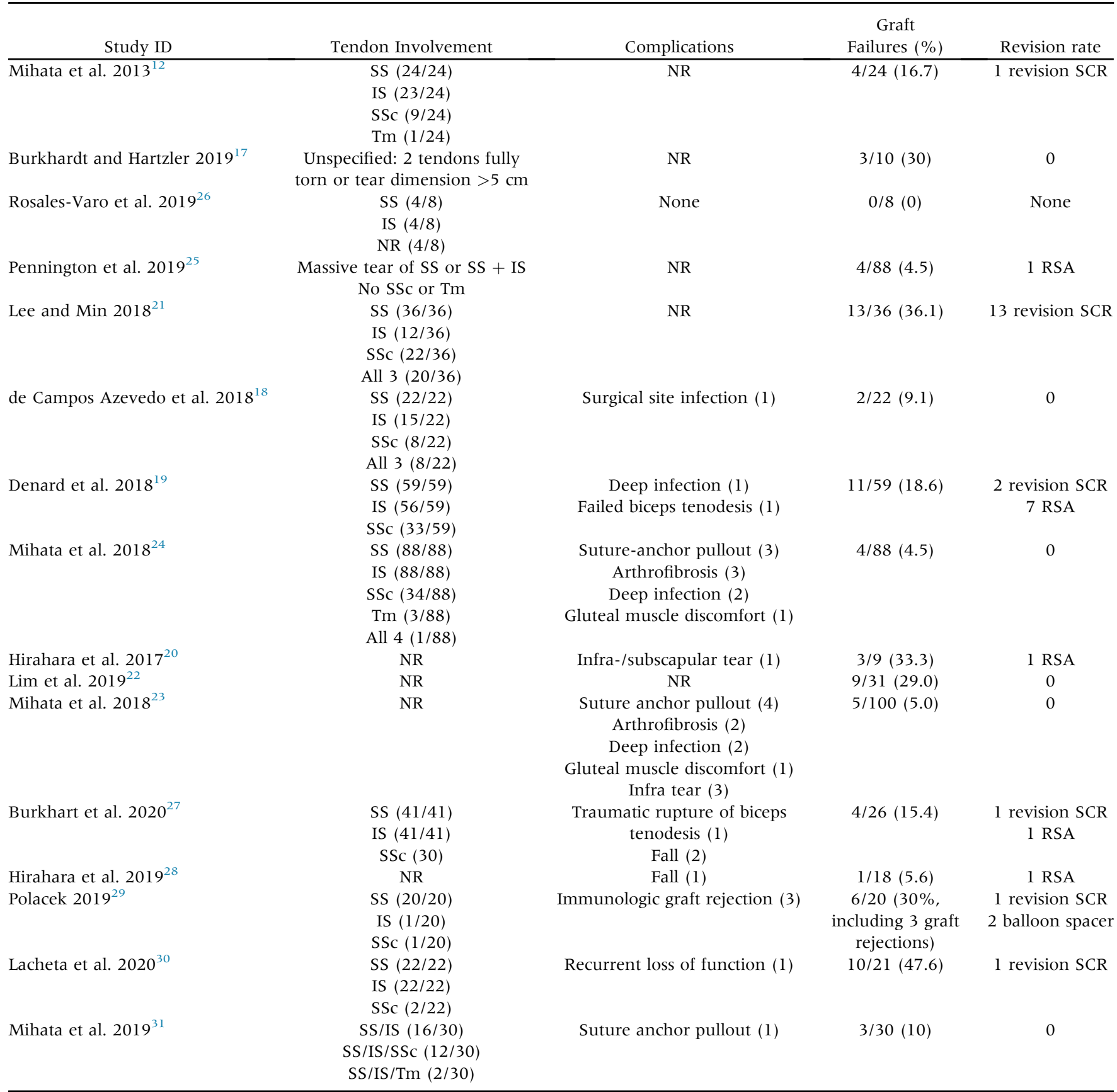

IS, infraspinatus; NR, not reported; RSA, reverse shoulder arthroplasty; SCR, superior capsular reconstruction; SS, supraspinatus; SSc, subscapularis; Tm, teres minor.

assessment. Although no level I or II studies currently exist on the topic, the low level of evidence limits the ability to pool data and provide a true quantitative analysis, and therefore also limits the overall strength of our conclusions. Fourth, the mean follow-up for our clinical studies is 36.9 months. Although this is a relatively short follow-up period, the longest follow-up of any study included is 60 months. Finally, a significant portion of the studies in this review were performed by small groups of researchers. This may introduce bias and falsely elevate the number of patients included in this review, as some patients may have been included in multiple studies.

\section{Conclusion}

Irrespective of tissue source, SCR serves as a reasonable joint-preserving option for massive, irreparable rotator cuff tears with favorable short- to midterm 
improvements in patient-reported outcomes and range of motion.

\section{References}

1. Gerber C, Wirth SH, Farshad M. Treatment options for massive rotator cuff tears. J Shoulder Elbow Surg 201 1;20: S20-S29.

2. Gerber C, Fuchs B, Hodler J. The results of repair of massive tears of the rotator cuff. J Bone Joint Surg Am 2000;82:505-515.

3. Goutallier D, Postel JM, Bernageau J, Lavau L, Voisin MC. Fatty muscle degeneration in cuff ruptures. Pre- and postoperative evaluation by CT scan. Clin Orthop Relat Res 1994:78-83.

4. Goutallier D, Postel J-M, Gleyze P, Leguilloux P, Van Driessche S. Influence of cuff muscle fatty degeneration on anatomic and functional outcomes after simple suture of full-thickness tears. J Shoulder Elbow Surg 2003;12:550-554.

5. Bedi A, Dines J, Warren RF, Dines DM. Massive tears of the rotator cuff. J Bone Joint Surg Am 2010;92:1894-1908.

6. Galatz LM, Ball CM, Teefey SA, Middleton WD, Yamaguchi K. The outcome and repair integrity of completely arthroscopically repaired large and massive rotator cuff tears. J Bone Joint Surg Am 2004;86:21 9-224.

7. Palsis JA, Simpson KN, Matthews JH, Traven S, Eichinger JK, Friedman RJ. Current trends in the use of shoulder arthroplasty in the United States. Orthopedics 2018;41:e416-e423.

8. Zumstein MA, Pinedo M, Old J, Boileau P. Problems, complications, reoperations, and revisions in reverse total shoulder arthroplasty: A systematic review. J Shoulder Elbow Surg 2011;20:146-157.

9. Ek ETH, Neukom L, Catanzaro S, Gerber C. Reverse total shoulder arthroplasty for massive irreparable rotator cuff tears in patients younger than 65 years old: Results after five to fifteen years. J Shoulder Elbow Surg 2013;22:1199-1208.

10. Ernstbrunner L, Suter A, Catanzaro S, Rahm S, Gerber C. Reverse total shoulder arthroplasty for massive, irreparable rotator cuff tears before the age of 60 years: Longterm results. J Bone Joint Surg Am 2017;99:1721-1729.

11. Adams CR, DeMartino AM, Rego G, Denard PJ, Burkhart SS. The rotator cuff and the superior capsule: Why we need both. Arthroscopy 2016;32:2628-2637.

12. Mihata T, Lee TQ, Watanabe C, et al. Clinical results of arthroscopic superior capsule reconstruction for irreparable rotator cuff tears. Arthroscopy 2013;29:459-470.

13. Higgins JPT, Thompson SG, Deeks JJ, Altman DG. Measuring inconsistency in meta-analyses. BMJ 2003;327:557-560.

14. DerSimonian R, Laird N. Meta-analysis in clinical trials revisited. Contemp Clin Trials 2015;45:139-145 (pt A).

15. Sidik K, Jonkman JN. A note on variance estimation in random effects meta-regression. J Biopharm Stat 2005;15: 823-838.

16. Stuck AE, Rubenstein LZ, Wieland D. Bias in metaanalysis detected by a simple, graphical test. Asymmetry detected in funnel plot was probably due to true heterogeneity. BMJ 1998;316:469.

17. Burkhart SS, Hartzler RU. Superior capsular reconstruction reverses profound pseudoparalysis in patients with irreparable rotator cuff tears and minimal or no glenohumeral arthritis. Arthroscopy 2019;35:22-28.

18. de Campos Azevedo CI, Ângelo ACLPG, Vinga S. Arthroscopic superior capsular reconstruction with a minimally invasive harvested fascia lata autograft produces good clinical results. Orthop J Sports Med 2018;6: 232596711880824.

19. Denard PJ, Brady PC, Adams CR, Tokish JM, Burkhart SS. Preliminary results of arthroscopic superior capsule reconstruction with dermal allograft. Arthroscopy 2018;34: 93-99.

20. Hirahara AM, Andersen WJ, Panero AJ. Superior capsular reconstruction: Clinical outcomes after minimum 2-year follow-up. Am J Orthop 2017;46:266-278.

21. Lee S-J, Min Y-K. Can inadequate acromiohumeral distance improvement and poor posterior remnant tissue be the predictive factors of re-tear? Preliminary outcomes of arthroscopic superior capsular reconstruction. Knee Surg Sports Traumatol Arthrosc 2018;26:2205-2213.

22. Lim S, AlRamadhan H, Kwak J-M, Hong H, Jeon I-H. Graft tears after arthroscopic superior capsule reconstruction (ASCR): Pattern of failure and its correlation with clinical outcome. Arch Orthop Trauma Surg 2019;139:231-239.

23. Mihata T, Lee TQ, Fukunishi K, et al. Return to sports and physical work after arthroscopic superior capsule reconstruction among patients with irreparable rotator cuff tears. Am J Sports Med 2018;46:1077-1083.

24. Mihata T, Lee TQ, Hasegawa A, et al. Arthroscopic superior capsule reconstruction can eliminate pseudoparalysis in patients with irreparable rotator cuff tears. Am J Sports Med 2018;46:2707-2716.

25. Pennington WT, Chen SW, Bartz BA, Pauli JM. Arthroscopic superior capsular reconstruction with acellular dermal allograft using push-in anchors for glenoid fixation. Arthrosc Tech 2019;8:e51-e55.

26. Rosales-Varo AP, Zafra M, García-Espona MA, FloresRuiz MA, Roda O. Superior capsular reconstruction of irreparable rotator cuff tear using autologous hamstring graft. Rev Esp Cir Ortop Traumatol 2019;63:1-6.

27. Burkhart SS, Pranckun JJ, Hartzler RU. Superior capsular reconstruction for the operatively irreparable rotator cuff tear: Clinical outcomes are maintained 2 years after surgery. Arthroscopy 2020;36:373-380.

28. Hirahara AM, Andersen WJ, Panero AJ. Ultrasound assessment of the superior capsular reconstruction with dermal allograft: An evaluation of graft thickness and vascularity. Arthroscopy 2019;35:3194-3202.

29. Polacek M. Arthroscopic superior capsular reconstruction with acellular porcine dermal xenograft for the treatment of massive irreparable rotator cuff tears. Arthrosc Sports Med Rehab 2019;1:e75-e84.

30. Lacheta L, Horan MP, Schairer WW, et al. Clinical and imaging outcomes after arthroscopic superior capsule reconstruction with human dermal allograft for irreparable posterosuperior rotator cuff tears: A minimum 2-year follow-up. Arthroscopy 2020;36:1011-1019.

31. Mihata T, Lee TQ, Hasegawa A, et al. Five-year follow-up of arthroscopic superior capsule reconstruction for irreparable rotator cuff tears. J Bone Joint Surg Am 2019;101:1921 - 1930.

32. Ferreira Neto AA, Malavolta EA, Assunção JH, Trindade EM, Gracitelli MEC. Reverse shoulder 
arthroplasty: Clinical results and quality of life evaluation. Rev Bras Ortop 2017;52:298-302.

33. Galvin JW, Kenney R, Curry EJ, et al. Superior capsular reconstruction for massive rotator cuff tears: A critical analysis review. JBJS Rev 2019;7:el.

34. Sochacki KR, McCulloch PC, Lintner DM, Harris JD. Superior capsular reconstruction for massive rotator cuff tear leads to significant improvement in range of motion and clinical outcomes: A systematic review. Arthroscopy 2019;35:1269-1277.

35. Catapano M, de SA D, Ekhtiari S, Lin A, Bedi A, Lesniak BP. Arthroscopic superior capsular reconstruction for massive, irreparable rotator cuff tears: A systematic review of modern literature. Arthroscopy 2019;35:1243-1253.

36. AlRamadhan H, Sungjoon L, In-Ho J. Early MRI findings of arthroscopic superior capsule reconstruction (ASCR): How to prevent early failure. Arthroscopy 2017;33:e21.

37. Zerr J, McDermott JD, Beckmann NM, Fullick RK, Chhabra A. Case study: Failure of superior capsular reconstruction using dermal allograft. Skelet Radiol 2017;46: 1585-1589.

38. Tokish JM, Momaya A, Roberson T. Superior capsular reconstruction with a partial rotator cuff repair: A case report. JBJS Case Connect 2018;8:e1.

39. Nimura A, Kato A, Yamaguchi K, et al. The superior capsule of the shoulder joint complements the insertion of the rotator cuff. J Shoulder Elbow Surg 2012;21:867-872.
40. Mihata T, Bui CNH, Akeda M, et al. A biomechanical cadaveric study comparing superior capsule reconstruction using fascia lata allograft with human dermal allograft for irreparable rotator cuff tear. J Shoulder Elbow Surg 2017;26:2158-2166.

41. Mihata T, McGarry MH, Kahn T, Goldberg I, Neo M, Lee TQ. Biomechanical effect of thickness and tension of fascia lata graft on glenohumeral stability for superior capsule reconstruction in irreparable supraspinatus tears. Arthroscopy 2016;32:418-426.

42. Makovicka JL, Chung AS, Patel KA, Deckey DG, Hassebrock JD, Tokish JM. Superior capsule reconstruction for irreparable rotator cuff tears: A systematic review of biomechanical and clinical outcomes by graft type. J Shoulder Elbow Surg September 2020;29:392-401.

43. Emerson CP, Balazs GC, Lee SC, Dines JS, Jose J, Greditzer HG. Magnetic resonance imaging of the failed superior capsular reconstruction. Clin Imaging 2020;60; 172-176.

44. Badman BL, Moor M, Baessler A, Crackel B. Short term comparative imaging and clinical analysis of superior capsular reconstruction. J Shoulder Elbow Surg 2020;29: el39-el40.

45. LaBelle MW, Peck M, Mengers S, et al. Evaluating the role of graft integrity on outcomes: Clinical and imaging results following superior capsular reconstruction. J Shoulder Elbow Surg 2020;29:e140-e141. 


\section{Appendix}

\section{Methods and Results for MINORS Criteria Scoring}

Methods-Quality Assessment

The Methodological Index for Non-randomized Studies (MINORS) checklist was used to assess the methodologic quality of included studies. The checklist assigns a score of $0-2$ for 8 items applicable to nonrandomized studies, with a maximum score of 16 indicating the highest possible score for an individual non-randomized study. The items were scored 0 if not reported; 1 when reported but inadequate; and 2 when reported and adequate. A minimum of 12 months was deemed appropriate length of follow-up. After thorough review of MINORS scoring guidelines, 2 authors independently reviewed and scored each included study. Any disagreements in scoring were resolved by consensus discussion with the senior author.

\section{Results-Study Quality}

The included studies were composed of 15 case series and 1 retrospective comparative study. All clinical studies were non-randomized. The mean MINORS score was 12.4 of 16. 